\title{
RKKY Interactions and Low-Temperature Behaviour of Spins in Quasiperiodic Tilings
}

\author{
S. THIEM* \\ Theoretical Physics, Oxford University, 1 Keble Road, Oxford OX1 3NP, UK
}

\begin{abstract}
We study the structure of the Ruderman-Kittel-Kasuya-Yosida interactions for selected quasiperiodic tilings. The interaction energies between the magnetic impurities in these systems are computed by a continued fraction expansion for the Green function of the conduction electrons. Based on these results we study the alignment of the magnetic moments in the Ammann-Beenker tiling by Monte Carlo simulations. In particular, we are interested in the structure of the magnetic ground state and the low-temperature behaviour for the Ising model.
\end{abstract}

DOI: $10.12693 /$ APhysPolA.126.613

PACS: $75.50 . \mathrm{Kj}, 61.43 . \mathrm{Bn}$

\section{Introduction}

Understanding the magnetic properties of complex materials still poses many open questions. In the case of quasicrystals this resulted in a controversial debate about the mechanisms of the formation and the alignment of magnetic moments in quasiperiodic atomic structures $[1,2]$. Regarding the second issue, several theoretical models predicted the occurrence of long-range magnetic order, but experimental results revealed a spin-glass-like behaviour at low temperatures [1].

In this paper we study the magnetic properties of simple models of rare-earth quasicrystals. According to experimental results the dominant interactions in rare-earth quasicrystals are due to the Ruderman-KittelKasuya-Yosida (RKKY) mechanism. This results in a long-range indirect exchange interaction between the localized rare-earth atoms which is mediated by the conduction electrons. First, we compute the RKKY interactions for the Ammann-Beenker tiling and the Rauzy tiling (see Sect. 2) using a continued fraction expansion for the Green function of the conduction electrons. This approach is able to consider the special properties of the eigenstates (multifractality) and the resulting anomalous diffusion behaviour which is usually observed for the electronic transport in quasicrystals. Based on these results we study the alignment of the magnetic moments for different temperatures and the magnetic ground state with parallel tempering (see Sect. 3).

To the best of our knowledge, long-range RKKY interactions and their influence on the alignment of magnetic moments have not been addressed in detail for quasiperiodic systems so far. Matsuo et al. studied the alignment of spins in a quasiperiodic $\mathrm{Zn}-\mathrm{Mg}-\mathrm{Ho}$ model and showed that the spin orientations can show long-range order and reflect the underlying quasiperiodic structure.

e-mail: stefanie.thiem@physics.ox.ac.uk
However, their approach has two drawbacks: on the one hand they included only the first four nearest-neighbour interactions, and on the other hand they used the standard RKKY formula for periodic systems [3, 4]. However, this formula is not applicable to quasiperiodic systems, and it is not clear if RKKY interactions are comparable in periodic and quasiperiodic systems. There are a few other results for long-range magnetic interactions including models with an exponential decay of the exchange interaction [5] and dipole interactions [6]. These models also yield uncommon spin structures which usually reflect certain aspects of the quasiperiodic tiling and in certain cases even exhibit long-range order.

\section{Computation of RKKY interactions in aperiodic tilings}

In our model the conduction electrons are described by a tight-binding Hamiltonian $H_{\text {cond }}=\sum_{\langle l, m\rangle}|l\rangle\langle m|$ with one orbital per site and equal hopping amplitudes between all nearest neighbors of the quasiperiodic tiling. In a dilute material the RKKY interactions between two magnetic impurities with spins $\sigma_{l}$ and $\sigma_{m}$ at the positions $l$ and $m$ are given by

$$
H_{\mathrm{RKKY}}=\lambda^{2} \chi_{l, m}\left(E_{\mathrm{F}}\right) \sigma_{l} \sigma_{m} .
$$

The factor $\lambda$ is proportional to the coupling of the magnetic moment to the conduction electrons, and the magnetic susceptibility [7] for zero temperature is

$$
\begin{aligned}
& \chi_{l, m}(E)=-\frac{1}{\pi} \int_{-\infty}^{\infty} \Im\left[G_{l, m}^{0}(E) G_{m, l}^{0}(E)\right] \\
& \quad \times \operatorname{sgn}\left(E_{\mathrm{F}}-E\right) \mathrm{d} E .
\end{aligned}
$$

Hence, the RKKY interactions depend on the Fermi energy $E_{\mathrm{F}}$ and the quasiperiodic structure, where the latter is taken into account by the two Green functions. In particular, $G_{l, m}^{0}(E)=\left\langle l\left|G^{0}(E)\right| m\right\rangle$ is the retarded Green function of the unperturbed problem, i.e., for the conduction electrons without the perturbation due to the two magnetic impurities.

There are several possibilities to compute the Green functions. This includes direct diagonalization, which 
is however limited to relatively small sample sizes and hence not well suited to study quasiperiodic tilings. Further, Roche and Mayou used an expansion of the Green function $G_{l, m}^{0}(E)$ in the Chebyshev polynomials [8]. This method has the drawback of producing artefacts for spiky density of states (DOS) of the conduction electrons which is a common feature in quasicrystals [7]. We will apply a third method which is based on the continued fraction expansion of the Green functions.

\subsection{Continued fraction expansion}

The continued fraction expansion for a Hamiltonian is a well-known tool in condensed matter physics and it was already used to compute the RKKY interactions for quasiperiodic tilings $[7,9]$. To obtain the susceptibility in Eq. (2) we first compute the local Green function of the conduction electrons, which can be expressed as a continued fraction

$$
G_{l, l}(E)=\frac{1}{E-a_{1}-\frac{b_{1}}{E-a_{2}-\frac{b_{2}}{E-a_{3}-\ldots}}} .
$$

The terms $a_{n}$ and $b_{n}$ are obtained by the tridiagonalization of the Hamiltonian $H_{\text {cond }}$. Due to the finite size of the system only a limited number of tridiagonalization steps are possible. To take the infinite environment into account, we approximate the tiling beyond a certain (fairly large) distance from the impurity by an average structure [10]. This yields for an infinite system with a gapless and symmetric DOS the values $a_{\infty}=0$ and $b_{\infty}=E_{\max }^{2} / 4$, i.e., $b_{\infty}=4.48$ for the Ammann-Beenker tiling [7] and $b_{\infty}=4.24$ for the two-dimensional Rauzy tiling.

In a second step the non-local Green functions $G_{l, m}^{0}(E)$ in Eq. (2) can be obtained from three local Green's functions [11] according to

$$
\begin{aligned}
& G_{l, m}^{0}(E)=(1+\mathrm{i})\left\langle\Psi^{+}\left|G^{0}(E)\right| \Psi^{+}\right\rangle+(-1+\mathrm{i}) \\
& \quad \times\left\langle\Psi^{-}\left|G^{0}(E)\right| \Psi^{-}\right\rangle-2 \mathrm{i}\left\langle\Psi^{\mathrm{i} m}\left|G^{0}(E)\right| \Psi^{\mathrm{i} m}\right\rangle,
\end{aligned}
$$

where $\left|\Psi^{ \pm}\right\rangle=|l\rangle \pm|m\rangle$ and $\left|\Psi^{\mathrm{i} m}\right\rangle=|l\rangle+\mathrm{i}|m\rangle$.

The continued fraction approach is an approximation technique which yields a smoothed version of the Green functions and hence the susceptibility [7]. With respect to real-world problems this approach is still reasonable because disorder and finite temperatures also lead to a smoothing of the sharp peaks of the Green functions in quasiperiodic tilings.

\section{2. $R K K Y$ interactions for Ammann-Beenker tiling and Rauzy tiling}

In this section we present the results for the structure of the RKKY interactions for two quasiperiodic tilings (see Fig. 1): (a) the Ammann-Beenker tiling, which is related to octagonal quasicrystals and possesses 6 different local environments with 3 to 8 nearest neighbours, and (b) the Rauzy tiling, which is a codimension one tiling and has $d+1$ different local environments in $d$ dimensions. To take the high degree of order in stable quasicrystals into account, we assume that the magnetic rare-earth atoms are localized at certain local environments (i.e. the same number of nearest neighbours).

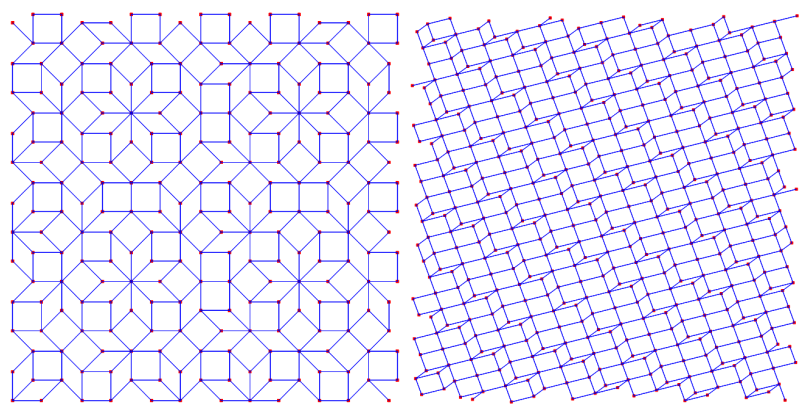

Fig. 1. Approximant of the Ammann-Beenker tiling with 239 sites (left) and of the Rauzy tiling with 504 sites (right).

The radial distribution $\chi(r)$ of the magnetic susceptibility is shown for the Ammann-Beenker tiling in Fig. 2. There is a clear dependence of the interaction strength on the Fermi energy and on the type of the local environment. Already the local DOS is significantly different for these cases [7], where - as a rule of thumb - stronger interactions can be found for higher local DOS at the Fermi energy. While we find only very small interactions strengths for local environments with 6,7 , or 8 nearest neighbours, we observe strong oscillations between ferroand antiferromagnetic interactions depending on the distances of the two impurities (in particular for $E_{\mathrm{F}}=0$ ) for environments with 3, 4, and 5 neighbours. Further, the Ammann-Beenker tiling for $E_{\mathrm{F}}=0$ corresponds to a bipartite lattice with half-filling. For such systems the RKKY interactions are antiferromagnetic $(\chi>0)$ for sites on the opposite sublattices and ferromagnetic $(\chi<0)$ for the same sublattice [12]. This is in excellent agreement with the susceptibility results in Fig. 2.
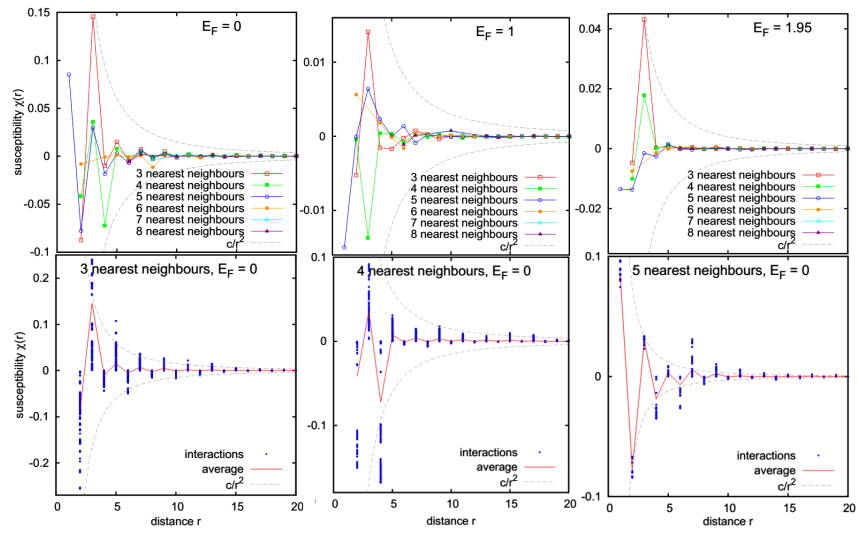

Fig. 2. Magnetic susceptibility for approximant $a=4$ of the Ammann-Beenker tiling with 1391 sites: average magnetic susceptibility for different local environments and Fermi energies $E_{\mathrm{F}}$ (top) and a comparison of averaged and non-averaged data for $E_{\mathrm{F}}=0$ (bottom). 
Additionally, the total exchange interaction $\chi_{\text {total }}=$ $\frac{1}{N} \sum_{l, m} \chi_{l, m}$ provides a rough measure of the preferred type of interaction. For the Ammann-Beenker tiling ferromagnetic interactions $\left(\chi_{\text {total }}<0\right)$ are dominant for 4 nearest neighbours and antiferromagnetic interactions $\left(\chi_{\text {total }}>0\right)$ for environments with 3 and 5 nearest neighbours. The exchange interactions are also rather consistent with different approximants despite the different system sizes. For a comparison we also included in Fig. 2 the expected interaction strength $\chi(r) \propto 1 / r^{d}$ for a periodic system in $d$ dimensions. While the average behaviour of the exchange interactions for the quasiperiodic systems is reasonably well described by this envelope, the single interactions can be considerably larger (see bottom row of Fig. 2) and we usually observe a whole hierarchy of interaction strengths.

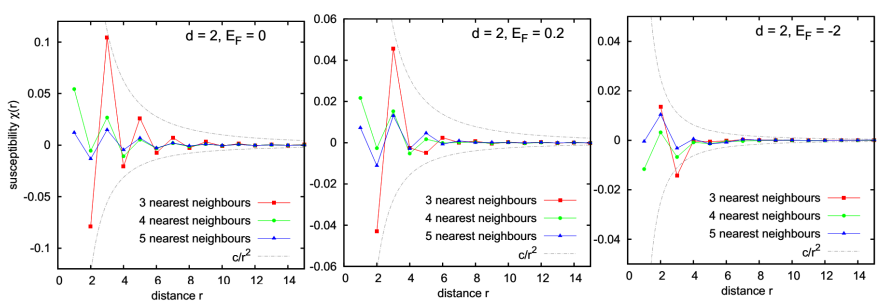

Fig. 3. Same as top row of Fig. 2 for the approximant $a=12$ of the two-dimensional Rauzy tiling with 548 sites.

Furthermore, we plot in Fig. 3 the magnetic susceptibility $\chi(r)$ for the two-dimensional Rauzy tiling. The results are very consistent with the previous results, i.e., $\chi(r)$ oscillates between ferro- and antiferromagnetic interactions and depends on the Fermi energy $E_{\mathrm{F}}$. As the system is relatively close to a periodic system (codimension of 1), the data is in quite good agreement with the envelope $\chi(r) \propto 1 / r^{d}$ for the periodic system.

\section{Monte Carlo simulations}

To study the alignment of magnetic moments we use Monte Carlo simulations for the Ising spins $\left(\sigma_{i}=\right.$ \pm 1 ) with quasiperiodic RKKY interactions according to Eq. (1). Many quasicrystals exhibit spin-glass freezing for low temperatures. Hence, it is of interest whether this behaviour is associated with the quasiperiodic order or with disorder and if there are differences in comparison to conventional spin glasses in frustrated disordered systems. According to the standard spin-glass theory, a spin-glass transition for the Ising model can be observed for long-range interactions with $\left\langle J^{2}\right\rangle=\left\langle\lambda^{4} \chi^{2}\right\rangle \propto 1 / r^{\alpha}$ and $\alpha<2 d$, i.e., RKKY interactions in periodic systems represent the limit case and are expected to show a zero-temperature spin-glass transition in two dimensions $[13,14]$. However, the RKKY interactions obtained in Sect. 2.2 show stronger oscillations, which could result in a transition at finite temperature in two dimensions.

\subsection{Parallel tempering}

A standard method to study magnetic moments in frustrated systems is parallel tempering. To overcome local minima of the energy landscape this approach uses $M$ copies (replicas) of the system at different temperatures $T_{M}=\frac{1}{\beta_{m}}$ (in the Planck units) $[15,16]$. We use a geometric distribution of the temperatures with $T_{m}=$ $c^{m} T_{\min }$ and $c=\left(T_{\max } / T_{\min }\right)^{1 /(M+1)}$. For each replica one Monte Carlo sweep (MCS) consists of $N$ single-spin flip attempts which are accepted with the probability $p=\max \{1, \exp (-\beta \Delta E)\}$ depending on the energy difference $\Delta E=E_{\text {new }}-E_{\text {old }}$ of the configurations. Further, the states of replicas with neighbouring temperatures are swapped with the probability $p_{\text {swap }}=\max \{1, \exp (-\Delta)\}$ with $\Delta=\left(\beta_{m+1}-\beta_{m}\right)\left(E_{m}-E_{m+1}\right)$. The number of replicas is chosen in a way to have a reasonably high acceptance ratio for the replica swaps. The benefit of parallel tempering compared to standard Monte Carlo methods is a significant reduction of the correlation times between local minima due to the simulation of the system at different temperatures.

We study the behaviour of various observables for spin systems including the mean magnetization $\langle M\rangle=\frac{1}{N}\left\langle\sum_{l=1}^{N} \sigma_{l}\right\rangle$, the mean staggered magnetization $\left\langle M^{\text {stag }}\right\rangle=\frac{1}{N}\left\langle\sum_{l=1}^{N} \eta_{l} \sigma_{l}\right\rangle$ with $\eta_{l}= \pm 1$ depending on the sublattice and the mean energy $\langle E\rangle=$ $\frac{1}{N}\left\langle\sum_{l, m} \chi_{l, m} \sigma_{l} \sigma_{m}\right\rangle$ per spin. The thermal average of an observable $O$ for $S$ MCS is given by $\langle O\rangle=\frac{1}{S} \sum_{i=1}^{S} O_{i}$. We equilibrate the systems for at least $3 \times 10^{5} \mathrm{MCS}$ and compute the averages from the last $10^{5}$ MCS. In the context of spin glasses an important quantity is the overlap field $q=\frac{1}{N} \sum_{l=1}^{N} \sigma_{l}^{1} \sigma_{l}^{2}$, which is obtained from the simulations of two independent replicas with the same temperature. Spin-glass transitions can be studied by the Binder cumulant $B=\frac{1}{2}\left(3-\left\langle q^{4}\right\rangle /\left\langle q^{2}\right\rangle^{2}\right)$ and the spin-glass susceptibility $\chi_{\mathrm{sg}}=N\left\langle q^{2}\right\rangle$.

\subsection{Low-temperature behaviour of spins}

In Fig. 4 we present the simulation results for the Ising spins with different local environments in the AmmannBeenker tiling. The divergence of the spin-glass susceptibility $\chi_{\mathrm{sg}}$ as well as the changes of the mean energy $\langle E\rangle$ and the Binder cumulant $B$ clearly indicate a phase transition from a paramagnetic state at high temperatures to a low-temperature state. The transition temperatures are $\frac{T}{\lambda^{2}} \approx 1.2$ for 4 nearest neighbours and $\frac{T}{\lambda^{2}} \approx 0.5$ for 5 nearest neighbours. The transition also results in a change of the overlap distribution.

We are in particular interested in the structure of the low-temperature spin states. The spin configurations with lowest energy (found during a simulation run) show in both cases local patterns of spins which repeat quasiperiodically (see Fig. 4). In the case of 4 nearest neighbours this pattern consists of 8 spins on the corners of the 8-fold stars which are pointing either all up or down. For 5 nearest neighbours the main pattern is 


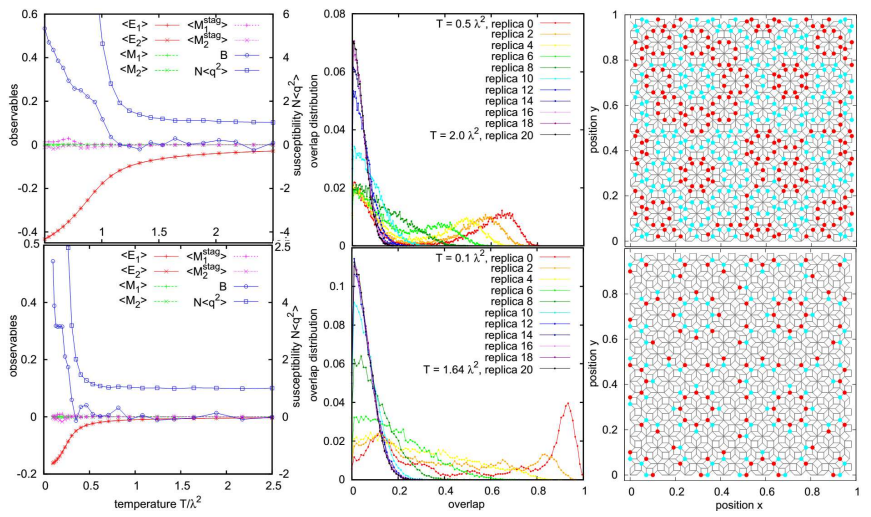

Fig. 4. Parallel tempering results for the AmmannBeenker tiling for sites with 4 (top) and 5 (bottom) nearest neighbours: different observables obtained from the two replicas (left), overlap distribution (center), and spin configuration for the lowest energy state (right).

an 8-fold double ring for which the spins of the outer and inner shell are aligned antiferromagnetically. These local spin patterns can be found repeatedly in the system because any local pattern of linear dimension $L$ in a quasiperiodic tiling is repeated in a distance $\mathcal{O}(L)$. Hence, the low-temperature state does not have a long-range periodic order but instead spins freeze in some hidden antiferromagnetic order. The low-temperature state also shows similarities to spin glasses (e.g. the occurrence of multiple ground states), where here the glassiness is caused by the quasiperiodicity instead of disorder.

\section{Conclusion}

The computation of RKKY interactions for quasiperiodic tilings revealed significant differences compared to periodic systems. Although we observe for both cases an oscillation between ferro- and antiferromagnetic interactions depending on the distance of the impurities, the range of the interaction in quasiperiodic tilings is often significantly larger than expected for a periodic system. By applying parallel tempering to study the alignment of magnetic moments for different spin distributions in the Ammann-Beenker tiling, we observe a phase transition towards a low-temperature state showing properties of a quasiperiodic antiferromagnetic state as well as a spin glass. This low-temperate state is characterized by specific local patterns which repeat quasiperiodically in the system.

In future we plan to study of alignment of spins with parallel tempering also for larger approximants and other quasiperiodic tilings including approximants of real quasicrystals.

\section{Acknowledgments}

The author would like to thank John Chalker for valuable discussions and helpful comments. The research was supported by the German Academic Exchange Service.

\section{References}

[1] F. Hippert, J.J. Prejean, Philos. Mag. 88, 2175 (2008).

[2] Z.M. Stadnik, in: Handbook of Magnetic Materials, Vol. 21, Ed. K.H.J. Buschow, Elsevier, NorthHolland, Amsterdam 2013.

[3] S. Matsuo, T. Ishimasa, H. Nakano, J. Magn. Magn. Mater. 246, 223 (2002).

[4] S. Matsuo, A. Aimurula, T. Ishimasa, S. Motomura, H. Nakano, Philos. Mag. 86, 741 (2006).

[5] E.Y. Vedmedenko, U. Grimm, R. Wiesendanger, Philos. Mag. 86, 733 (2006).

[6] E.Y. Vedmedenko, H.P. Oepen, J. Kirschner, Phys. Rev. Lett. 90, 137203 (2003).

[7] A. Jagannathan, J. Phys. I (France) 4, 133 (1994).

[8] S. Roche, D. Mayou, Phys. Rev. B 60, 322 (1999).

[9] V. Kumar, V, G. Athithan, Phys. Rev. B 35, 906 (1987).

[10] R. Haydock, V. Heine, M.J. Kelly, J. Phys. C, Solid State Phys. 8, 2591 (1975).

[11] F. Triozon, S. Roche, Eur. Phys. J. B 46, 427 (2005).

[12] S. Saremi, Phys. Rev. B 76, 184430 (2007).

[13] A.J. Bray, M.A. Moore, A.P. Young, Phys. Rev. Lett. 56, 2641 (1986).

[14] H.G. Katzgraber, A.P. Young, Phys. Rev. B 67, 134410 (2003).

[15] R.H. Swendsen, J.S. Wang, Phys. Rev. Lett. 57, 2607 (1986).

[16] E. Marinari, G. Parisi, Europhys. Lett. 19, 451 (1992). 